\section{TATrA \\ MOUNTaiNS \\ Mathematical Publications}

DOI: $10.2478 / \mathrm{tmmp}-2021-0021$

Tatra Mt. Math. Publ. 79 (2021), 81-100

\title{
OSCILLATION TESTS \\ FOR LINEAR DIFFERENCE EQUATIONS WITH NON-MONOTONE ARGUMENTS
}

\author{
George E. Chatzarakis*1 — SAid R. Grace ${ }^{2}$ - Irena Jadlovská ${ }^{3}$ \\ ${ }^{1}$ Department of Electrical and Electronic Engineering Educators, School of Pedagogical and \\ Technological Education (ASPETE), Athens, GREECE \\ ${ }^{2}$ Department of Engineering Mathematics, Faculty of Engineering, Cairo University, Orman, Giza, \\ EGYPT \\ ${ }^{3}$ Department of Mathematics and Theoretical Informatics, Faculty of Electrical Engineering and \\ Informatics Technical University of Košice, Košice, SLOVAKIA
}

ABSTRACT. This paper presents sufficient conditions involving lim sup for the oscillation of all solutions of linear difference equations with general deviating argument of the form

$$
\Delta x(n)+p(n) x(\tau(n))=0, n \in \mathbb{N}_{0} \quad[\nabla x(n)-q(n) x(\sigma(n))=0, n \in \mathbb{N}],
$$

where

$$
(p(n))_{n \geq 0} \text { and }(q(n))_{n \geq 1}
$$

are sequences of nonnegative real numbers and

$$
(\tau(n))_{n \geq 0}, \quad(\sigma(n))_{n \geq 1}
$$

are (not necessarily monotone) sequences of integers. The results obtained improve all well-known results existing in the literature and an example, numerically solved in MATLAB, illustrating the significance of these results is provided.

\section{Introduction}

In this paper we consider the difference equation with a variable deviating argument of either retarded

$$
\Delta x(n)+p(n) x(\tau(n))=0, \quad n \in \mathbb{N}_{0}
$$

or advanced type

$$
\nabla x(n)-q(n) x(\sigma(n))=0, \quad n \in \mathbb{N} .
$$

(C) 2021 Mathematical Institute, Slovak Academy of Sciences.

2010 Mathematics Subject Classification: 39A10, 39A21.

Keywords: non-monotone argument, retarded argument, advanced argument, oscillation, Grönwall inequality.

(c) (1) ()요 Licensed under the Creative Commons BY-NC-ND 4.0 International Public License. 
Equations $(\mathbb{E})$ and $\left(\mathbb{E}^{\prime}\right)$ are studied under the following assumptions: everywhere

$$
3(p(n))_{n \geq 0} \text { and }(q(n))_{n \geq 1}
$$

are sequences of nonnegative real numbers, and

$$
(\tau(n))_{n \geq 0}, \quad(\sigma(n))_{n \geq 1}
$$

are sequences of integers satisfying

$$
\tau(n) \leq n-1, \quad \forall n \in \mathbb{N}_{0} \quad \text { and } \quad \lim _{n \rightarrow \infty} \tau(n)=\infty
$$

and

$$
\sigma(n) \geq n+1, \quad \forall n \in \mathbb{N},
$$

respectively. Here, $\Delta$ denotes the forward difference operator $\Delta x(n)=x(n+$ 1) $-x(n)$ and $\nabla$ corresponds to the backward difference operator $\nabla x(n)=$ $x(n)-x(n-1)$.

Set

$$
w=-\min _{n \geq 0} \tau(n) .
$$

Clearly, $w$ is a finite positive integer if (1.1) holds.

By a solution of $(\mathrm{E})$, we mean a sequence of real numbers $(x(n))_{n>-w}$ which satisfies (E) for all $n \geq 0$. By a solution of $\left(\mathrm{E}^{\prime}\right)$, we mean a sequence of real numbers $(x(n))_{n \geq 0}$ which satisfies $\left(\mathrm{E}^{\prime}\right)$ for all $n \geq 1$.

A solution $(x(n))_{n>-w}\left(\right.$ or $\left.(x(n))_{n>0}\right)$ of $(\underline{E})\left(\right.$ or $\left(\mathbb{E}^{\prime}\right)$ is called oscillatory, if the terms $x(n)$ of the sequence are neither eventually positive nor eventually negative. Otherwise, the solution is said to be nonoscillatory. An equation is oscillatory if all its solutions oscillate.

It is clear that, for each choice of real numbers $c_{-w}, c_{-w+1}, \ldots, c_{-1}, c_{0}$, by using the method of steps one can obtain a unique solution $(x(n))_{n>-w}$ to (E) satisfying the initial conditions $x(-w)=c_{-w}, x(-w+1)=c_{-w+1}, \ldots$, $x(-1)=c_{-1}, x(0)=c_{0}$.

It is noteworthy to observe that a first-order linear difference equation of the form $\left.(\mathbb{E})\left[\mathbb{E}^{\prime}\right)\right]$ without retarded $(\tau(n) \equiv n)$ [advanced $\left.(\sigma(n) \equiv n)\right]$ argument does not possess oscillatory solutions. Therefore, the investigation of oscillatory solutions is of interest for equations of the form $(\underline{E})\left[\left(\mathrm{E}^{\prime}\right)\right]$. Furthermore, the mathematical modelling of several real-world problems leads to difference equations that depend on the past or future state values rather than only the current one.

The problem of establishing sufficient conditions for the oscillation of all solutions of equations $(\mathrm{E})$ and $\left(\mathrm{E}^{\prime}\right)$ has been the subject of many investigations, see, for example, [1-[16] and the references cited therein. Most of these papers concerned with the special case where the arguments are nondecreasing, while a 
few of these papers are dealing with the general case where the arguments are not necessarily monotone. See, for example, [1]-[5], [8]- 9].

The consideration of non-monotone arguments other than the pure mathematical interest, it approximates the natural phenomena described by equation of the type $(\mathrm{E})$ or $\left(\mathrm{E}^{\prime}\right)$. That is because there are always natural disturbances (e.g., noise in communication systems) that affect all the parameters of the equation and therefore the fair (from a mathematical point of view) monotone arguments become almost always non-monotone. In view of this, an interesting question arising in the case where the argument $\tau(n)[\sigma(n)]$ is non-monotone, is whether we can state further oscillation criteria which essentially improve all the known results in the literature. In the present paper, a positive answer to the above mentioned question is given.

The paper is organized as follows. First, we present, separately for a retarded and advanced case, some of the related results which motivate the contents of this paper. Next, we establish new sufficient conditions, involving limsup, for the oscillation of all solutions of $(\mathrm{E})$ and $\left(\mathrm{E}^{\prime}\right)$. We base our technique on the proper use of an iterative procedure leading to new inequalities which may replace former ones. To verify the significance of the results, we provide an example along with various comparisons among new and known criteria.

Throughout this paper, we are going to use the following notation:

$$
\begin{aligned}
\sum_{i=k}^{k-1} A(i)=0 \quad \text { and } \quad & \prod_{i=k}^{k-1} A(i)=1, \\
\alpha:=\liminf _{n \rightarrow \infty} \sum_{j=\tau(n)}^{n-1} p(j) \quad \text { and } \quad & \beta:=\liminf _{n \rightarrow \infty} \sum_{j=n+1}^{\sigma(n)} q(j),
\end{aligned}
$$

and

$$
D(\omega):= \begin{cases}0 & \text { if } \omega>1 / e, \\ \frac{1-\omega-\sqrt{1-2 \omega-\omega^{2}}}{2} & \text { if } \omega \in[0,1 / e] .\end{cases}
$$

\subsection{Retarded difference equations}

In 2008, Chatzarakis, Koplatadze and Stavroulakis [6, 7] proved that, if $\tau(n)$ is nondecreasing and

or

$$
\limsup _{n \rightarrow \infty} \sum_{j=\tau(n)}^{n} p(j)>1
$$

$$
\liminf _{n \rightarrow \infty} \sum_{j=\tau(n)}^{n-1} p(j)>\frac{1}{e},
$$




\section{GEORGE E. CHATZARAKIS-SAID R. GRACE-IRENA JADLOVSKÁ}

then all solutions of $(\mathrm{E})$ are oscillatory.

It is obvious that there is a gap between the conditions (1.5) and (1.6) when the limit

$$
\lim _{n \rightarrow \infty} \sum_{j=\tau(n)}^{n-1} p(j)
$$

does not exist. How to fill this gap is an interesting problem which has been investigated by several authors.

Now we come to the case that the argument $\tau(n)$ is not necessarily monotone. Set

$$
h(n)=\max _{0 \leq s \leq n} \tau(s) .
$$

Clearly, the sequence $h(n)$ is nondecreasing with $\tau(n) \leq h(n) \leq n-1$ for all $n \geq 0$.

In 2011, Braverman and Karpuz [2] proved that, if

$$
\limsup _{n \rightarrow \infty} \sum_{j=h(n)}^{n} p(j) \prod_{i=\tau(j)}^{h(n)-1} \frac{1}{1-p(i)}>1
$$

then all solutions of (E) are oscillatory.

In 2015, Braverman et al [1] proved that, if for some $r \in \mathbb{N}$

$$
\limsup _{n \rightarrow \infty} \sum_{j=h(n)}^{n} p(j) a_{r}^{-1}(h(n), \tau(j))>1,
$$

where

$$
\begin{aligned}
a_{1}(n, k) & =\prod_{i=k}^{n-1}[1-p(i)], \\
a_{r+1}(n, k) & =\prod_{i=k}^{n-1}\left[1-p(i) a_{r}^{-1}(i, \tau(i))\right],
\end{aligned}
$$

then all solutions of (E) are oscillatory.

Remark 1. Observe that condition (1.8) is a special case of (1.9), when $r=1$.

Several improvements were made to the above conditions, see [3]-[5], 8], 9] to arrive at the recent forms 3

$$
\limsup _{n \rightarrow \infty} \sum_{k=h(n)}^{n} p(k) \exp \left(\sum_{\ell=\tau(k)}^{h(n)-1} p(\ell) \exp \left(\sum_{j=\tau(\ell)}^{\ell-1} p(j) \prod_{u=\tau(j)}^{j-1} \frac{1}{1-d_{\xi}(u)}\right)\right)>1
$$


where

$$
\begin{aligned}
& d_{\xi}(n) \\
& =p(n)\left[1+\sum_{k=\tau(n)}^{n-1} p(k) \exp \left(\sum_{\ell=\tau(k)}^{n-1} p(\ell) \exp \left(\sum_{j=\tau(\ell)}^{\ell-1} p(j) \prod_{u=\tau(j)}^{j-1} \frac{1}{1-d_{\xi-1}(u)}\right)\right)\right]
\end{aligned}
$$

with

$$
d_{0}(n)=p(n)\left[1+\sum_{k=\tau(n)}^{n-1} p(k) \exp \left(\sum_{\ell=\tau(k)}^{n-1} p(\ell) \exp \left(\lambda_{0} \sum_{j=\tau(\ell)}^{\ell-1} p(j)\right)\right]\right.
$$

and $\lambda_{0}$ is the smaller root of the transcendental equation $\lambda=e^{\alpha \lambda}$.

\subsection{Advanced difference equations}

In 2012, Chatzarakis and Stavroulakis [10] proved that, if $\sigma(n)$ is nondecreasing and

$$
\limsup _{n \rightarrow \infty} \sum_{j=n}^{\sigma(n)} q(j)>1
$$

then all solutions of $\left(\mathrm{E}^{\prime}\right)$ are oscillatory.

Now we come to the case that the argument $\sigma(n)$ is not necessarily monotone. Set

$$
\rho(n)=\min _{s \geq n} \sigma(s) .
$$

Clearly, the sequence $\rho(n)$ is nondecreasing with $\sigma(n) \geq \rho(n) \geq n+1$ for all $n \geq 1$.

In 2015, Braverman et al [1] proved that, if for some $r \in \mathbb{N}$

$$
\limsup _{n \rightarrow \infty} \sum_{j=n}^{\rho(n)} q(j) b_{r}^{-1}(\rho(n), \sigma(j))>1
$$

where

$$
\begin{gathered}
b_{1}(n, k)=\prod_{i=n+1}^{k}[1-q(i)], \\
b_{r+1}(n, k)=\prod_{i=n+1}^{k}\left[1-q(i) b_{r}^{-1}(i, \sigma(i))\right],
\end{gathered}
$$

then all solutions of $\left(\mathrm{E}^{\prime}\right.$ are oscillatory. 
Several improvements were made to the above conditions, see $[3-5,8,9]$ to arrive at the recent forms [3]

$$
\limsup _{n \rightarrow \infty} \sum_{k=n}^{\rho(n)} q(k) \exp \left(\sum_{\ell=\rho(n)+1}^{\sigma(k)} q(\ell) \exp \left(\sum_{j=\ell+1}^{\sigma(\ell)} q(j) \prod_{u=j+1}^{\sigma(j)} \frac{1}{1-g_{\xi}(u)}\right)\right)>1
$$

where

$$
\begin{aligned}
& g_{\xi}(n) \\
& =q(n)\left[1+\sum_{k=n+1}^{\sigma(n)} q(k) \exp \left(\sum_{\ell=n+1}^{\sigma(k)} q(\ell) \exp \left(\sum_{j=\ell+1}^{\sigma(\ell)} q(j) \prod_{u=j+1}^{\sigma(j)} \frac{1}{1-g_{\xi-1}(u)}\right)\right)\right]
\end{aligned}
$$

with

$$
g_{0}(n)=q(n)\left[1+\sum_{k=n+1}^{\sigma(n)} q(k) \exp \left(\sum_{\ell=n+1}^{\sigma(k)} q(\ell) \exp \left(\lambda_{0} \sum_{j=\ell+1}^{\sigma(\ell)} q(j)\right)\right]\right.
$$

and $\lambda_{0}$ is the smaller root of the transcendental equation $\lambda=e^{\beta \lambda}$.

\section{Main results}

\subsection{Retarded difference equations}

We further study (E) and derive new sufficient oscillation conditions, involving lim sup, which improve on all previously known results in the literature.

The proofs of our main results are essentially based on the following lemmas.

Lemma 1 (See 9]). Assume that $h(n)$ is defined by (1.7). If $\alpha>0$ then

$$
\liminf _{n \rightarrow \infty} \sum_{j=h(n)}^{n-1} p(j)=\liminf _{n \rightarrow \infty} \sum_{j=\tau(n)}^{n-1} p(j)=\alpha .
$$

Lemma 2 (See [4]). Assume that $h(n)$ is defined by (1.7) and $x(n)$ is an eventually positive solution of (E). If $0<\alpha \leq 1 / e$ then

$$
\liminf _{n \rightarrow \infty} \frac{x(n+1)}{x(h(n))} \geq D(\alpha)
$$

and

$$
\liminf _{n \rightarrow \infty} \frac{x(h(n))}{x(n)} \geq \lambda_{0},
$$

where $\lambda_{0}$ is the smaller root of the transcendental equation $\lambda=e^{\alpha \lambda}$. 
Theorem 1. Assume that (1.1) holds and $h(n)$ is defined by (1.7). If for some $\varphi \in \mathbb{N}$

$$
\limsup _{n \rightarrow \infty} \sum_{s=h(n)}^{n} p(s) \exp \left(\sum_{j=\tau(s)}^{h(n)-1} p(j) \prod_{m=\tau(j)}^{j-1} \frac{1}{1-b_{\varphi}(m)}\right)>1,
$$

where

$$
b_{\varphi}(n)=p(n)\left[1+\sum_{s=\tau(n)}^{n-1} p(s) \exp \left(\sum_{u=\tau(s)}^{n-1} p(u) \prod_{i=\tau(u)}^{u-1} \frac{1}{1-b_{\varphi-1}(i)}\right)\right]
$$

with

$$
\begin{gathered}
b_{0}(n)=p(n)\left[1+\sum_{m=\tau(n)}^{n-1} p(m) \exp \left(\sum_{\ell=\tau(m)}^{n-1} p(\ell) \exp \left(S_{k}(\ell)\right)\right)\right], \\
S_{k}(n)=\sum_{j_{k}=\tau(n)}^{n-1} p\left(j_{k}\right) \exp \left(S_{k-1}\left(j_{k}\right)\right), \quad k \geq 1, \quad \text { with } \quad S_{0}(n)=\lambda_{0} \sum_{j_{0}=\tau(n)}^{n-1} p\left(j_{0}\right),
\end{gathered}
$$

and $\lambda_{0}$ is the smaller root of the transcendental equation $\lambda=e^{\alpha \lambda}$, then all solutions of (E) are oscillatory.

P r o o f. Assume, for the sake of contradiction, that $(x(n))_{n \geq-w}$ is a nonoscillatory solution of (E). Then it is either eventually positive or eventually negative. As $(-x(n))_{n>-w}$ is also a solution of $(\mathrm{E})$, we may restrict ourselves only to the case where $x(n)>0$ for all large $n$. Let $n_{1} \geq-w$ be an integer such that $x(n)>0$ for all $n \geq n_{1}$. Then, there exists $n_{2} \geq n_{1}$ such that $x(\tau(n))>0$, $\forall n \geq n_{2}$. In view of this, Eq. (E) becomes

$$
\Delta x(n)=-p(n) x(\tau(n)) \leq 0, \quad \forall n \geq n_{2},
$$

which means that the sequence $(x(n))$ is eventually nonincreasing.

Dividing (E) by $x(n)$ and summing up from $\tau(n)$ to $n-1$, we take

$$
\sum_{j_{0}=\tau(n)}^{n-1} \frac{\Delta x\left(j_{0}\right)}{x\left(j_{0}\right)}=-\sum_{j_{0}=\tau(n)}^{n-1} p\left(j_{0}\right) \frac{x\left(\tau\left(j_{0}\right)\right)}{x\left(j_{0}\right)} .
$$


Also, since $e^{x} \geq x+1, x \in \mathbb{R}$, we have

$$
\begin{aligned}
\sum_{j_{0}=\tau(n)}^{n-1} \frac{\Delta x\left(j_{0}\right)}{x\left(j_{0}\right)} & =\sum_{j_{0}=\tau(n)}^{n-1}\left(\frac{x\left(j_{0}+1\right)}{x\left(j_{0}\right)}-1\right) \\
& =\sum_{j_{0}=\tau(n)}^{n-1}\left[\exp \left(\ln \frac{x\left(j_{0}+1\right)}{x\left(j_{0}\right)}\right)-1\right] \\
& \geq \sum_{j_{0}=\tau(n)}^{n-1}\left[\ln \frac{x\left(j_{0}+1\right)}{x\left(j_{0}\right)}+1-1\right] \\
& =\sum_{j_{0}=\tau(n)}^{n-1} \ln \frac{x\left(j_{0}+1\right)}{x\left(j_{0}\right)}=\ln \frac{x(n)}{x(\tau(n))},
\end{aligned}
$$

or

$$
\sum_{j_{0}=\tau(n)}^{n-1} \frac{\Delta x\left(j_{0}\right)}{x\left(j_{0}\right)} \geq \ln \frac{x(n)}{x(\tau(n))} .
$$

Combining (2.5) and (2.6), we obtain

$$
-\sum_{j_{0}=\tau(n)}^{n-1} p\left(j_{0}\right) \frac{x\left(\tau\left(j_{0}\right)\right)}{x\left(j_{0}\right)} \geq \ln \frac{x(n)}{x(\tau(n))},
$$

or

$$
\ln \frac{x(\tau(n))}{x(n)} \geq \sum_{j_{0}=\tau(n)}^{n-1} p\left(j_{0}\right) \frac{x\left(\tau\left(j_{0}\right)\right)}{x\left(j_{0}\right)}
$$

Thus

$$
\begin{aligned}
x(\tau(n)) & \geq x(n) \exp \left(\sum_{j_{0}=\tau(n)}^{n-1} p\left(j_{0}\right) \frac{x\left(\tau\left(j_{0}\right)\right)}{x\left(j_{0}\right)}\right) \\
& \geq x(n) \exp \left(\sum_{j_{0}=\tau(n)}^{n-1} p\left(j_{0}\right) \frac{x\left(h\left(j_{0}\right)\right)}{x\left(j_{0}\right)}\right) \\
& \geq x(n) \exp \left(\left(\lambda_{0}-\epsilon\right) \sum_{j_{0}=\tau(n)}^{n-1} p\left(j_{0}\right)\right)
\end{aligned}
$$

or

$$
x(\tau(n)) \geq x(n) \exp \left(S_{0}(n, \epsilon)\right),
$$

where

$$
S_{0}(n, \epsilon)=\left(\lambda_{0}-\epsilon\right) \sum_{j_{0}=\tau(n)}^{n-1} p\left(j_{0}\right) .
$$


Combining (E) and (2.7), we have

$$
\Delta x(n)+p(n) x(n) \exp \left(S_{0}(n, \epsilon)\right) \leq 0 .
$$

Dividing (2.8) by $x(n)$ and summing up from $\tau(n)$ to $n-1$, we take

$$
\sum_{j_{1}=\tau(n)}^{n-1} \frac{\Delta x\left(j_{1}\right)}{x\left(j_{1}\right)} \leq-\sum_{j_{1}=\tau(n)}^{n-1} p\left(j_{1}\right) \exp \left(S_{0}\left(j_{1}, \epsilon\right)\right)
$$

or

$$
x(\tau(n)) \geq x(n) \exp \left(\sum_{j_{1}=\tau(n)}^{n-1} p\left(j_{1}\right) \exp \left(S_{0}\left(j_{1}, \epsilon\right)\right)\right)=x(n) \exp \left(S_{1}(n, \epsilon)\right)
$$

where

$$
S_{1}(n, \epsilon)=\sum_{j_{1}=\tau(n)}^{n-1} p\left(j_{1}\right) \exp \left(S_{0}\left(j_{1}, \epsilon\right)\right)
$$

Continuing by induction, for sufficiently large $n$, we can construct the following:

$$
x(\tau(n)) \geq x(n) \exp \left(S_{k}(n, \epsilon)\right), \quad(k \in \mathbb{N}),
$$

where

$$
S_{k}(n, \epsilon)=\sum_{j_{k}=\tau(n)}^{n-1} p\left(j_{k}\right) \exp \left(S_{k-1}\left(j_{k}, \epsilon\right)\right) .
$$

Combining (E) and (2.10), we have

$$
\Delta x(n)+p(n) x(n) \exp \left(S_{k}(n, \epsilon)\right) \leq 0 .
$$

We divide (2.12) by $x(n)>0$ and summing up on $[\tau(m), n-1]$ we have

$$
\sum_{\ell=\tau(m)}^{n-1} \frac{\Delta x(\ell)}{x(\ell)} \leq-\sum_{\ell=\tau(m)}^{n-1} p(\ell) \exp \left(S_{k}(\ell, \epsilon)\right)
$$

or

$$
x(\tau(m)) \geq x(n) \exp \left(\sum_{\ell=\tau(m)}^{n-1} p(\ell) \exp \left(S_{k}(\ell, \epsilon)\right)\right) .
$$

Summing up (E) from $\tau(n)$ to $n-1$, using (2.13) and multiplying by $p(n)$, we obtain

$$
\begin{aligned}
p(n) x(n)-p(n) x(\tau(n))+p(n) x(n) \times & \\
& \sum_{m=\tau(n)}^{n-1} p(m) \exp \left(\sum_{\ell=\tau(m)}^{n-1} p(\ell) \exp \left(S_{k}(\ell, \epsilon)\right)\right) \leq 0
\end{aligned}
$$

which, in view of (E), becomes 


$$
\begin{aligned}
& \Delta x(n)+p(n) x(n)+p(n) x(n) \times \\
& \sum_{m=\tau(n)}^{n-1} p(m) \exp \left(\sum_{\ell=\tau(m)}^{n-1} p(\ell) \exp \left(S_{k}(\ell, \epsilon)\right)\right) \leq 0 .
\end{aligned}
$$

Thus

$$
\Delta x(n)+p(n)\left[1+\sum_{m=\tau(n)}^{n-1} p(m) \exp \left(\sum_{\ell=\tau(m)}^{n-1} p(\ell) \exp \left(S_{k}(\ell, \epsilon)\right)\right)\right] x(n) \leq 0,
$$

or

$$
\Delta x(n)+b_{0}(n, \epsilon) x(n) \leq 0
$$

with

$$
b_{0}(n, \epsilon)=p(n)\left[1+\sum_{m=\tau(n)}^{n-1} p(m) \exp \left(\sum_{\ell=\tau(m)}^{n-1} p(\ell) \exp \left(S_{k}(\ell, \epsilon)\right)\right)\right] .
$$

Applying the discrete Grönwall inequality in (2.14), we obtain

$$
x(\tau(u))>x(u) \prod_{i=\tau(u)}^{u-1} \frac{1}{1-b_{0}(i, \epsilon)} .
$$

Dividing (E) by $x(n)$, summing up from $\tau(s)$ to $n-1$ and in view of (2.15), we take

$$
\begin{aligned}
\ln \frac{x(n)}{x(\tau(s))} & \leq \sum_{u=\tau(s)}^{n-1} \frac{\Delta x(u)}{x(u)}=-\sum_{u=\tau(s)}^{n-1} p(u) \frac{x(\tau(u))}{x(u)} \\
& \leq-\sum_{u=\tau(s)}^{n-1} p(u) \prod_{i=\tau(u)}^{u-1} \frac{1}{1-b_{0}(i, \epsilon)}
\end{aligned}
$$

or

$$
x(\tau(s)) \geq x(n) \exp \left(\sum_{u=\tau(s)}^{n-1} p(u) \prod_{i=\tau(u)}^{u-1} \frac{1}{1-b_{0}(i, \epsilon)}\right) .
$$

Summing up (E) from $\tau(n)$ to $n-1$ and using (2.16), we obtain

$$
x(n)-x(\tau(n))+\sum_{s=\tau(n)}^{n-1} p(s) x(\tau(s))=0 .
$$

Combining (2.16) and (2.17), we find

$$
\begin{aligned}
& x(n)-x(\tau(n))+x(n) \times \\
& \sum_{s=\tau(n)}^{n-1} p(s) \exp \left(\sum_{u=\tau(s)}^{n-1} p(u) \prod_{i=\tau(u)}^{u-1} \frac{1}{1-b_{0}(i, \epsilon)}\right) \leq 0,
\end{aligned}
$$


Multiplying the last inequality by $p(n)$, we get

$$
\begin{aligned}
p(n) x(n)-p(n) x(\tau(n))+p(n) x(n) \times & \\
& \sum_{s=\tau(n)}^{n-1} p(s) \exp \left(\sum_{u=\tau(s)}^{n-1} p(u) \prod_{i=\tau(u)}^{u-1} \frac{1}{1-b_{0}(i, \epsilon)}\right)<0,
\end{aligned}
$$

which, in view of (E), becomes

$$
\begin{aligned}
\Delta x(n)+p(n) x(n)+ & p(n) x(n) \times \\
& \sum_{s=\tau(n)}^{n-1} p(s) \exp \left(\sum_{u=\tau(s)}^{n-1} p(u) \prod_{i=\tau(u)}^{u-1} \frac{1}{1-b_{0}(i, \epsilon)}\right)<0,
\end{aligned}
$$

i.e.,

$$
\Delta x(n)+p(n)\left[1+\sum_{s=\tau(n)}^{n-1} p(s) \exp \left(\sum_{u=\tau(s)}^{n-1} p(u) \prod_{i=\tau(u)}^{u-1} \frac{1}{1-b_{0}(i, \epsilon)}\right)\right] x(n)<0 .
$$

Therefore

$$
\Delta x(n)+b_{1}(n, \epsilon) x(n)<0,
$$

where

$$
b_{1}(n, \epsilon)=p(n)\left[1+\sum_{s=\tau(n)}^{n-1} p(s) \exp \left(\sum_{u=\tau(s)}^{n-1} p(u) \prod_{i=\tau(u)}^{u-1} \frac{1}{1-b_{0}(i, \epsilon)}\right)\right] .
$$

Repeating the above argument leads to a new estimate

$$
\Delta x(n)+b_{2}(n, \epsilon) x(n)<0,
$$

where

$$
b_{2}(n, \epsilon)=p(n)\left[1+\sum_{s=\tau(n)}^{n-1} p(s) \exp \left(\sum_{u=\tau(s)}^{n-1} p(u) \prod_{i=\tau(u)}^{u-1} \frac{1}{1-b_{1}(i, \epsilon)}\right)\right] .
$$

Continuing by induction, for sufficiently large $n$ we get

$$
\Delta x(n)+b_{\varphi}(n, \epsilon) x(n) \leq 0,
$$

where

$$
b_{\varphi}(n, \epsilon)=p(n)\left[1+\sum_{s=\tau(n)}^{n-1} p(s) \exp \left(\sum_{u=\tau(s)}^{n-1} p(u) \prod_{i=\tau(u)}^{u-1} \frac{1}{1-b_{\varphi-1}(i, \epsilon)}\right)\right]
$$

and

$$
x(\tau(s))>x(h(n)) \exp \left(\sum_{j=\tau(s)}^{h(n)-1} p(j) \prod_{m=\tau(j)}^{j-1} \frac{1}{1-b_{\varphi}(m, \epsilon)}\right) .
$$


Summing up (E) from $h(n)$ to $n$, we have

$$
x(n+1)-x(h(n))+\sum_{s=h(n)}^{n} p(s) x(\tau(s))=0 .
$$

Combining (2.20) and (2.21), we have, for all sufficiently large $n$,

$$
\begin{array}{r}
x(n+1)-x(h(n))+x(h(n)) \\
\sum_{s=h(n)}^{n} p(s) \exp \left(\sum_{j=\tau(s)}^{h(n)-1} p(j) \prod_{m=\tau(j)}^{j-1} \frac{1}{1-b_{\varphi}(m, \epsilon)}\right)<0 .
\end{array}
$$

The inequality is valid if we omit $x(n+1)>0$ in the left-hand side

$$
\begin{array}{r}
-x(h(n))+x(h(n)) \times \\
\sum_{s=h(n)}^{n} p(s) \exp \left(\sum_{j=\tau(s)}^{h(n)-1} p(j) \prod_{m=\tau(j)}^{j-1} \frac{1}{1-b_{\varphi}(m, \epsilon)}\right)<0 .
\end{array}
$$

Thus, as $x(h(n))>0$, for all sufficiently large $n$ it holds

$$
\sum_{s=h(n)}^{n} p(s) \exp \left(\sum_{j=\tau(s)}^{h(n)-1} p(j) \prod_{m=\tau(j)}^{j-1} \frac{1}{1-b_{\varphi}(m, \epsilon)}\right)<1,
$$

from which by letting $n \rightarrow \infty$, we have

$$
\limsup _{n \rightarrow \infty} \sum_{s=h(n)}^{n} p(s) \exp \left(\sum_{j=\tau(s)}^{h(n)-1} p(j) \prod_{m=\tau(j)}^{j-1} \frac{1}{1-b_{\varphi}(m, \epsilon)}\right) \leq 1 .
$$

Since $\epsilon$ may be taken arbitrarily small, this inequality contradicts (2.3).

The proof of the theorem is complete.

Theorem 2. Assume that $h(n)$ is defined by (1.7) and $\alpha \in(0,1 / e]$. If for some $\varphi \in \mathbb{N}$

$$
\limsup _{n \rightarrow \infty} \sum_{s=h(n)}^{n} p(s) \exp \left(\sum_{j=\tau(s)}^{h(n)-1} p(j) \prod_{m=\tau(j)}^{j-1} \frac{1}{1-b_{\varphi}(m)}\right)>1-D(\alpha),
$$

where $b_{\varphi}$ is defined by (2.4), then all solutions of (E) are oscillatory.

Proof. Let $x$ be an eventually positive solution of Eq. (E). Then, as in the proof of Theorem 1, we obtain (2.22), i.e., for sufficiently large $n$ we have 


$$
\begin{aligned}
& x(n+1)-x(h(n))+x(h(n)) \times \\
& \sum_{s=h(n)}^{n} p(s) \exp \left(\sum_{j=\tau(s)}^{h(n)-1} p(j) \prod_{m=\tau(j)}^{j-1} \frac{1}{1-b_{\varphi}(m, \epsilon)}\right)<0 .
\end{aligned}
$$

Thus,

$$
\sum_{s=h(n)}^{n} p(s) \exp \left(\sum_{j=\tau(s)}^{h(n)-1} p(j) \prod_{m=\tau(j)}^{j-1} \frac{1}{1-b_{\varphi}(m, \epsilon)}\right)<1-\frac{x(n+1)}{x(h(n))},
$$

which gives

$$
\limsup _{n \rightarrow \infty} \sum_{s=h(n)}^{n} p(s) \exp \left(\sum_{j=\tau(s)}^{h(n)-1} p(j) \prod_{m=\tau(j)}^{j-1} \frac{1}{1-b_{\varphi}(m, \epsilon)}\right) \leq 1-\liminf _{t \rightarrow \infty} \frac{x(n+1)}{x(h(n))} .
$$

By Lemma 2, it is obvious that inequality (2.1) is fulfilled. So, the last inequality leads to

$$
\limsup _{n \rightarrow \infty} \sum_{s=h(n)}^{n} p(s) \exp \left(\sum_{j=\tau(s)}^{h(n)-1} p(j) \prod_{m=\tau(j)}^{j-1} \frac{1}{1-b_{\varphi}(m, \epsilon)}\right) \leq 1-D(\alpha) .
$$

Since $\epsilon$ may be taken arbitrarily small, this inequality contradicts (2.23).

The proof of the theorem is complete.

\subsection{Advanced difference equations}

Similar lemmas for the (dual) advanced difference equation $\left(\mathrm{E}^{\prime}\right.$, easily, can be derived. The proofs of these lemmas are omitted, since they are quite similar to those of the corresponding lemmas, for the retarded equation.

Lemma 3. Assume that $\rho(n)$ is defined by (1.13). If $\beta>0$ then

$$
\liminf _{n \rightarrow \infty} \sum_{j=n+1}^{\rho(n)} q(j)=\liminf _{n \rightarrow \infty} \sum_{j=n+1}^{\sigma(n)} q(j)=\beta .
$$

Lemma 4. Assume that $\rho(n)$ is defined by (1.13), $0<\beta \leq 1 / e$ and $x(n)$ is an eventually positive solution of ( $\left.\mathrm{E}^{\prime}\right)$. Then

$$
\liminf _{n \rightarrow \infty} \frac{x(n-1)}{x(\rho(n))} \geq D(\beta)
$$

and

$$
\liminf _{n \rightarrow \infty} \frac{x(\rho(n))}{x(n)} \geq \lambda_{0},
$$

where $\lambda_{0}$ is the smaller root of the transcendental equation $\lambda=e^{\beta \lambda}$. 


\section{GEORGE E. CHATZARAKIS—SAID R. GRACE—IRENA JADLOVSKÁ}

Based on Lemmas 3 and 4, we derive new sufficient oscillation conditions, involving limsup, which essentially improve all previously known results in the literature. The proofs of these theorems are omitted, since they are quite similar to those of the corresponding theorems, for the retarded equation.

Theorem 3. Assume that (1.2) holds and $\rho(n)$ is defined by (1.13). If for some $\varphi \in \mathbb{N}$

$$
\limsup _{n \rightarrow \infty} \sum_{s=n}^{\sigma(n)} q(s) \exp \left(\sum_{j=\rho(n)+1}^{\sigma(s)} q(j) \prod_{m=j+1 \tau(j)}^{\sigma(j)} \frac{1}{1-d_{\varphi}(m)}\right)>1,
$$

where

$$
d_{\varphi}(n)=q(n)\left[1+\sum_{s=n+1}^{\sigma(n)} q(s) \exp \left(\sum_{u=n+1}^{\sigma(s)} q(u) \prod_{i=u+1}^{\sigma(u)} \frac{1}{1-d_{\varphi-1}(i)}\right)\right]
$$

with

$$
\begin{gathered}
d_{0}(n)=q(n)\left[1+\sum_{m=n+1}^{\sigma(n)} q(m) \exp \left(\sum_{\ell=n+1}^{\sigma(m)} q(\ell) \exp \left(S_{k}(\ell)\right)\right)\right] \\
S_{k}(n)=\sum_{j_{k}=n+1}^{\sigma(n)} q\left(j_{k}\right) \exp \left(S_{k-1}\left(j_{k}\right)\right), k \geq 1 \quad \text { with } \quad S_{0}(n)=\lambda_{0} \sum_{j_{0}=n+1}^{\sigma(n)} q\left(j_{0}\right)
\end{gathered}
$$

and $\lambda_{0}$ is the smaller root of the transcendental equation $\lambda=e^{\beta \lambda}$, then all solutions of $\left(\mathrm{E}^{\prime}\right)$ are oscillatory.

Theorem 4. Assume that (1.2) holds, $\rho(n)$ is defined by (1.13) and $0<\beta \leq 1 / e$. If for some $\varphi \in \mathbb{N}$

$$
\limsup _{n \rightarrow \infty} \sum_{s=n}^{\sigma(n)} q(s) \exp \left(\sum_{j=\rho(n)+1}^{\sigma(s)} q(j) \prod_{m=j+1 \tau(j)}^{\sigma(j)} \frac{1}{1-d_{\varphi}(m)}\right)>1-D(\beta),
$$

where $d_{\varphi}$ is defined by (2.25), then all solutions of ( $\left.\mathrm{E}^{\prime}\right)$ are oscillatory.

\subsection{Difference inequalities}

We close Section 2 by citing some results concerning the corresponding to (E) or $\left(\mathrm{E}^{\prime}\right)$ difference inequalities. As the proofs of these results require only slight modifications in the proofs of Theorems 1, 4, we omit them. 
Theorem 5. Assume that all conditions of Theorem 1 (Theorem 3) or Theorem 2 (Theorem 4) hold. Then

(i) the retarded [advanced] difference inequality

$$
\Delta x(n)+p(n) x(\tau(n)) \leq 0, \quad n \in \mathbb{N}_{0} \quad[\nabla x(n)-q(n) x(\sigma(n)) \geq 0, n \in \mathbb{N}]
$$

has no eventually positive solutions;

(ii) the retarded [advanced] difference inequality

$$
\Delta x(n)+p(n) x(\tau(n)) \geq 0, \quad n \in \mathbb{N}_{0} \quad[\nabla x(n)-q(n) x(\sigma(n)) \leq 0, n \in \mathbb{N}]
$$

has no eventually negative solutions.

\section{An example and comments}

The example below illustrates that our conditions essentially improve known results in the literature yet indicate a type of independence among some of them. Not to pursue complexity any further, we choose to present an example with constant coefficients and variable non-monotone argument. This example does not only illustrate the significance of our results but also indicates a high level of improvement in the oscillation criteria. The calculations were made by the use of MATLAB software.

ExAmple 1 (taken and adapted from [3]). Consider the retarded difference equation

$$
\Delta x(n)+\frac{23}{250} x(\tau(n))=0, \quad n \in \mathbb{N}_{0},
$$

with (see Fig. 1, (a))

$$
\tau(n)= \begin{cases}n-1 & \text { if } n=5 \mu \\ n-6 & \text { if } n=5 \mu+1 \\ n-2 & \text { if } n=5 \mu+2, \\ n-6 & \text { if } n=5 \mu+3 \\ n-3 & \text { if } n=5 \mu+4,\end{cases}
$$

where $\mu \in \mathbb{N}_{0}$ and $\mathbb{N}_{0}$ is the set of nonnegative integers.

By (1.7), we see (Fig. 1, (b)) that

$$
h(n)= \begin{cases}n-1 & \text { if } n=5 \mu, \\ n-2 & \text { if } n=5 \mu+1, \\ n-2 & \text { if } n=5 \mu+2 \\ n-3 & \text { if } n=5 \mu+3 \\ n-3 & \text { if } n=5 \mu+4 .\end{cases}
$$




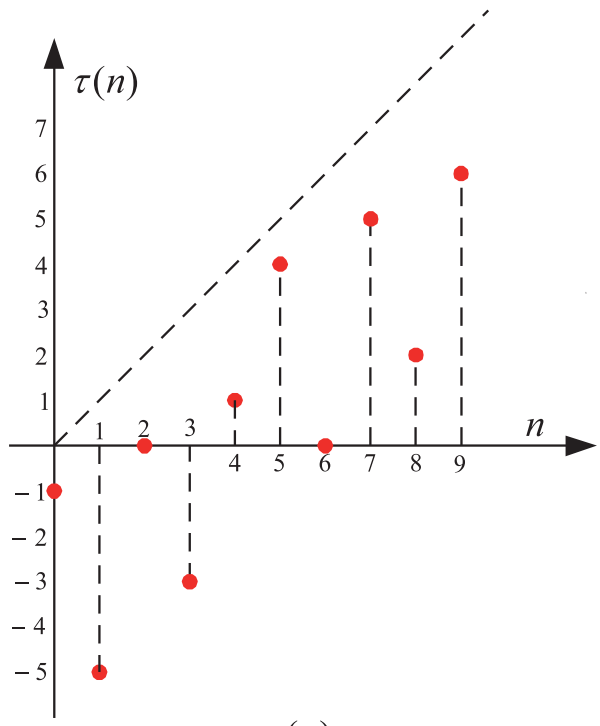

(a)

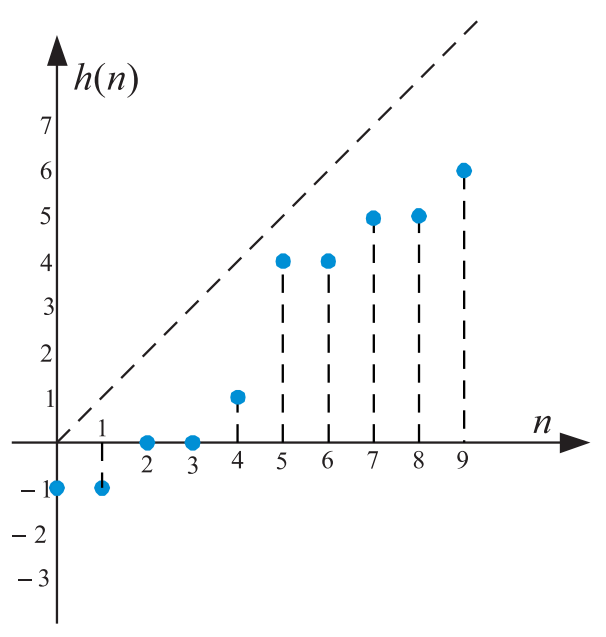

(b)

Figure 1. The graphs of $\tau(n)$ and $h(n)$.

It is easy to see that

$$
\alpha=\liminf _{n \rightarrow \infty} \sum_{j=\tau(n)}^{n-1} p(j)=\liminf _{\mu \rightarrow \infty} \sum_{j=5 \mu-1}^{5 \mu-1} \frac{23}{250}=0.092
$$

and therefore, the smaller root of $e^{0.092 \lambda}=\lambda$ is $\lambda_{0}=1.10723$.

Observe that the function $F_{\xi}: \mathbb{N}_{0} \rightarrow \mathbb{R}_{+}$defined as

$$
F_{\varphi}(n, k)=\sum_{s=h(n)}^{n} p(s) \exp \left(\sum_{j=\tau(s)}^{h(n)-1} p(j) \prod_{m=\tau(j)}^{j-1} \frac{1}{1-b_{\varphi}(m, \epsilon)}\right),
$$

attains its maximum at

$$
n=5 \mu+4, \quad \mu \in \mathbb{N}_{0}, \quad \text { for every } \varphi \in \mathbb{N} .
$$

Specifically, by using an algorithm implemented in MATLAB software, we obtain

$$
F_{1}(5 \mu+4,3)=\sum_{s=5 \mu+1}^{5 \mu+4} p(s) \exp \left(\sum_{j=\tau(s)}^{5 \mu} p(j) \prod_{m=\tau(j)}^{j-1} \frac{1}{1-b_{1}(m, \epsilon)}\right) \simeq 1.021
$$

and therefore

$$
\limsup _{n \rightarrow \infty} F_{1}(n, 3) \simeq 1.021>1 .
$$


That is, condition (2.3) of Theorem 1 is satisfied for $\varphi=1$. Therefore, all solutions of (3.1) are oscillatory.

Observe, however, that

$$
\begin{aligned}
& \limsup _{n \rightarrow \infty} \sum_{j=\tau(n)}^{n} p(j)=\limsup _{\mu \rightarrow \infty} \sum_{s=5 \mu+1}^{5 \mu+4} \frac{23}{250}=0.38<1, \\
& \alpha=0.092<\frac{1}{e} \\
& \limsup _{n \rightarrow \infty} \sum_{j=h(n)}^{n} p(j) \prod_{i=\tau(j)}^{h(n)-1} \frac{1}{1-p(i)} \\
& =\limsup _{\mu \rightarrow \infty} \sum_{j=5 \mu+1}^{5 \mu+4} \frac{23}{250} \prod_{i=\tau(j)}^{5 \mu} \frac{1}{1-\frac{23}{250}} \\
& =\frac{23}{250}\left[\frac{1}{\left(1-\frac{23}{250}\right)^{6}}+\frac{1}{1-\frac{23}{250}}+\frac{1}{\left(1-\frac{23}{250}\right)^{4}}+1\right] \\
& \simeq 0.4928<1 .
\end{aligned}
$$

Also, by using an algorithm in MATLAB software, we obtain

$$
\limsup _{n \rightarrow \infty} \sum_{k=h(n)}^{n} p(k) \exp \left(\sum_{\ell=\tau(k)}^{h(n)-1} p(\ell) \exp \left(\sum_{j=\tau(\ell)}^{\ell-1} p(j) \prod_{u=\tau(j)}^{j-1} \frac{1}{1-d_{1}(u)}\right)\right) \simeq 0.7177<1 \text {. }
$$

That is, none of conditions (1.5), (1.6), (1.8) $\equiv(1.9)$ (for $r=1)$ and (1.15) (for $\xi=1$ ) is satisfied.

Notation 1. It is worth noting that the improvement of condition (2.3) to the corresponding condition (1.5) is significant, approximately $168.68 \%$, if we compare the values on the left-side of these conditions. Also, the improvement compared to conditions (1.8) $\equiv(1.9)$ (for $r=1$ ) and (1.15) (for $\xi=1$ ) is very satisfactory, around $107.18 \%$ and $42.26 \%$, respectively.

Finally, observe that the conditions (1.9) and (1.15) do not lead to oscillation for the first iteration. On the contrary, condition (2.3) is satisfied from the first iteration. This means that our condition is better and much faster than (1.9) and (1.15).

Remark 2. Similarly, one can construct examples, illustrating the other main results, in the paper. 


\section{GEORGE E. CHATZARAKIS-SAID R. GRACE—IRENA JADLOVSKÁ}

\section{Concluding Remarks}

The recursive sequence $S_{k}(n)$ directly affects the sequence $b_{0}(n)$. We have been trying and still try to analytically compute the limit of $S_{k}(n)$, since there are strong indications that the limit exists.

This is because:

(1) The sequence $S_{k}(n)$ is bounded. Indeed, assume that $\left\{S_{k}(n)\right\}_{k \in \mathbb{N}}$ is unbounded. Then there exists a subsequence $S_{\theta(k)}(n) \rightarrow \infty$ as $k \rightarrow \infty$.

Since

$$
x(\tau(n)) \geq x(n) \exp \left(S_{k}(n, \epsilon)\right), \quad(k \in \mathbb{N}),
$$

it is obvious that

$$
x(\tau(n)) \geq x(n) \exp \left(S_{\theta(k)}(n, \epsilon)\right) x(\tau(t))
$$

or

$$
\frac{x(n)}{x(\tau(n))} \leq \exp \left(-S_{\theta(k)}(n, \epsilon)\right) \rightarrow 0 \text { as } k \rightarrow \infty .
$$

Consequently, $x(n)=0$, which contradicts $x(n)>0$.

(2) The indications (using MATLAB) give us the impression that $\left\{S_{k}(n)\right\}_{k \in \mathbb{N}}$ is increasing.

Therefore, this limit seems to exist. The question is whether this limit can be found (open problem), which is obviously a function of $p(n)$ and $\tau(n)$. However, we sent it for publication with major contribution the construction of the recursive sequence $\left\{S_{k}(n)\right\}_{k \in \mathbb{N}}$, which always allows the condition (2.3) to be the best of all the known oscillation conditions.

MATLAB calculations for this example show that there is a rapid convergence (approximately 7 repetitions $(n=7)$ are needed). However, the resulting value is approximately $1.4>>1$. This enables us to reduce the value of $p(n)$ of equation (E) in each repetition, since our goal is always to achieve the value $1^{+}$in the condition (2.3). In this way, all known conditions will not be satisfied in contrast to (2.3) which will be. However, the reduction of the value of $p(n)$ can be made up to a point, since if the condition

$$
\sum_{j=\tau(n)}^{n} p(j) \leq \frac{1}{e}
$$

holds eventually, then $(\mathbb{E})$ has a nonoscillatory solution.

Therefore, if the limit of $\left\{S_{k}(n)\right\}_{k \in \mathbb{N}}$ is not analytically found, we can always have the optimal condition with respect to all known oscillation conditions, but we can never define precisely the reduction of the value of $p(n)$ without violating the oscillation. 


\section{NON-MONOTONE ARGUMENTS}

However, the problem remains open, and we believe that finally we will be able to calculate the limit of the sequence $\left\{S_{k}(n)\right\}_{k \in \mathbb{N}}$.

Finishing, we think that if the limit exists but cannot be analytically computed, this manuscript is the best possible result that leads to oscillation when all the known conditions do not apply.

Similar comments can be made for equation ( $\left(\mathrm{E}^{\prime}\right)$.

\section{Disclosure statement}

No potential conflict of interest was reported by the authors.

\section{REFERENCES}

[1] BRAVERMAN, E.-CHATZARAKIS, G. E.-STAVROULAKIS, I. P.: Iterative oscillation tests for difference equations with several non-monotone arguments, J. Difference Equ. Appl. 21 (2015), no. 9, 854-874.

[2] E. BRAVERMAN, E.-KARPUZ, B.: On oscillation of differential and difference equations with non-monotone delays, Appl. Math. Comput. 218 (2011), 3880-3887.

[3] CHATZARAKIS, G. E.: Sufficient oscillation conditions for deviating difference equations, Filomat 33 (2019), no. 11, 3291-3305.

[4] CHATZARAKIS, G. E.-JADLOVSKÁ, I.: Oscillations in deviating difference equations using an iterative technique, J. Inequal. Appl. 2017, Paper No. 173, 24 pp.

[5] CHATZARAKIS, G. E.-JADLOVSKÁ, I.: Improved iterative oscillation tests for firstorder deviating difference equations, Int. J. Difference Equ. 12 (2017), no. 2, 185-210.

[6] CHATZARAKIS, G. E.-KOPLATADZE, R.-STAVROULAKIS, I P.: Oscillation criteria of first order linear difference equations with delay argument, Nonlinear Anal. 68 (2008), 994-1005.

[7] Chatzarakis, G. E.-R. KOPLATADZE, R.-STAVROULAKIS, I. P.: Optimal oscillation criteria for first order difference equations with delay argument, Pacific J. Math. 235 (2008), 15-33.

[8] CHATZARAKIS, G. E.-PURNARAS, I. K.-STAVROULAKIS, I. P.: Oscillations of deviating difference equations with non-monotone arguments, J. Difference Equ. Appl. 23 (2017), no. 8, 1354-1377.

[9] CHATZARAKIS, G.E.-SHAIKHET, L.: Oscillation criteria for difference equations with non-monotone arguments, Adv. Difference Equ. 2017, Paper No. 62, 16 pp.

[10] CHATZARAKIS, G. E.-STAVROULAKIS, I. P.: Oscillations of difference equations with general advanced argument, Cent. Eur. J. Math. 10 (2012), 807-823.

[11] CHEN, M.-P.-YU, J.S.: Oscillations of delay difference equations with variable coefficients. In: Proceedings of the First International Conference on Difference Equations, Gordon and Breach, London, 1994, pp. 105-114.

[12] LI, X.-ZHU, D.: Oscillation of advanced difference equations with variable coefficients, Ann. Differential Equations 18 (2002), 254-263.

[13] TANG, X. H.-YU, J. S.: Oscillation of delay difference equations, Comput. Math. Appl. 37 (1999), 11-20. 


\section{GEORGE E. CHATZARAKIS—SAID R. GRACE—IRENA JADLOVSKÁ}

[14] TANG, X.H.- ZHANG, R. Y.: New oscillation criteria for delay difference equations, Comput. Math. Appl. 42 (2001), 1319-1330.

[15] YAN, W.-MENG, Q.-YAN, J.: Oscillation criteria for difference equation of variable delays, DCDIS Proceedings 3 (2005), 641-647.

[16] ZHANG, B. G. - TIAN, C. J.: Nonexistence and existence of positive solutions for difference equations with unbounded delay, Comput. Math. Appl. 36 (1998), 1-8.

Received August 23, 2020

George E. Chatzarakis*

Department of Electrical and

Electronic Engineering Educators

School of Pedagogical and

Technological Education (ASPETE)

Marousi 15122, Athens

GREECE

E-mail: geaxatz@otenet.gr,gea.xatz@aspete.gr

Said R. Grace

Department of Engineering Mathematics

Faculty of Engineering

Cairo University, Orman

Giza 12221

EGYPT

E-mail: saidgrace@yahoo.com

Irena Jadlovská

Department of Mathematics and

Theoretical Informatics

Faculty of Electrical Engineering

and Informatics

Technical University of Košice

Letná 9

04200 Košice

SLOVAKIA

E-mail: irena.jadlovska@tuke.sk 